\section{Her tongue tipped us off...}

$\mathrm{A}_{\text {h }}^{1}$ 15-year-old previously healthy girl presented to a tertiary care pediatric hospital emergency department in the fall with a history of vague rightsided pleuritic chest pain and dizziness. For the previous few months, she had been experiencing anorexia (with a 7-kg weight loss), epigastric discomfort and fatigue. Upon further questioning, the patient's mother stated that her daughter's summer suntan had not faded even though she had spent most of the previous month indoors. In addition, she was drinking up to $3 \mathrm{~L}$ of water a day and craving salty foods and pickles. On physical examination, her blood pressure was 90/50 $\mathrm{mm} \mathrm{Hg}$ supine and 80/40 $\mathrm{mm} \mathrm{Hg}$ sitting, her heart rate was 100 beats/min, her respiratory rate 16 breaths/min, and she was afebrile. Her skin was tanned and she had areas of hyperpigmentation on her tongue (Fig. 1). She had a slightly low sodium level (133 mmol/L) and a high normal potassium level $(5.0 \mathrm{mmol} / \mathrm{L})$. She was euglycemic and had evidence of mild prerenal failure (creatinine level $123 \mu \mathrm{mol} / \mathrm{L}$ and urea level $10.7 \mathrm{mmol} / \mathrm{L})$. She had a high normal leukocyte count $(10.3 \times$ $10 \% / \mathrm{L})$ with an elevated eosinophil count $\left(1.34 \times 10^{9} / \mathrm{L}\right)$, mild anemia (hemoglobin $123 \mathrm{~g} / \mathrm{L}$ ) and a normal platelet count (362 $\left.\times 10^{\circ} / \mathrm{L}\right)$. Her erythrocyte sedimentation rate was elevated at $31 \mathrm{~mm} / \mathrm{h}$. A random cortisol level was low at $<25 \mathrm{nmol} / \mathrm{L}$, which, despite variation in normal values, is highly suggestive of adrenal insufficiency. She had a normal chest radiograph and electrocardiogram. She was given a normal saline bolus to treat her hypotension and admitted to $\circ$ hospital for further investigation. An adrenocorticotropin hormone (ACTH) stimulation test revealed serum cortisol levels of $25 \mathrm{nmol} / \mathrm{L}$ at baseline and $27 \mathrm{nmol} / \mathrm{L}$ at 60 minutes (normally 165-680 nmol/L in the morning; the peak cortisol level with an ACTH stimulation test should be > $500 \mathrm{nmol} / \mathrm{L})$. She was found to have primary adrenal insufficiency and given hydrocortisone and fludrocortisone. In hospital her symptoms improved, and her electrolyte level and renal function returned to normal.

Primary adrenal insufficiency, or Addison's disease, was first described by Thomas Addison in 1855. In North America it is most commonly caused by autoimmune destruction of the adrenal gland by antiadrenal autoantibodies. ${ }^{1}$ This condition can be either isolated or associated with a polyglandular autoimmune endocrinopathy of the parathyroid gland, thyroid gland or pancreas. ${ }^{2}$ The incidence of primary adrenal insufficiency is 4.7-6.2 per million in white populations, ${ }^{3}$ with peak incidence in the fourth decade; women are affected more frequently than men. ${ }^{2}$ Symptoms of Addison's disease are nonspecific and may mimic malignant disease as well as gastrointestinal, psychiatric and other endocrine disorders. Salt craving is a more specific symptom of Addison's disease and occurs in about $15 \%$ of cases. ${ }^{3}$ Hyperpigmentation, a relatively specific sign, occurs in $94 \%$ of cases ${ }^{4}$ and is most pronounced in areas that are exposed to increased friction (e.g., palmar creases, knuckles, scars and oral mucosa). This finding is caused by increased ACTH and melanocyte stimulating hormone secondary to the compensatory activation of the hypothalamicpituitary axis. Most patients who have adrenal insufficiency have a low random cortisol level at the time of diagnosis, but some may have a normal serum cortisol lev$\mathrm{el}$; in this case, an ACTH stimulation test is required to confirm the diagnosis. Mild anemia, lymphocytosis, eosinophilia and hypoglycemia may also occur. Mineralocorticoid deficiency causes

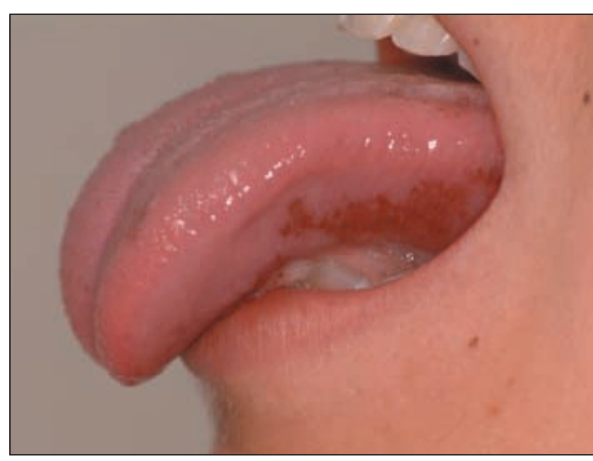

hyponatremia and hyperkalemia in $90 \%$ and $65 \%$ of patients respectively. ${ }^{3}$ Dehydration and prerenal failure may also occur.

Treatment of Addison's disease includes cortisol and mineralocorticoid replacement therapy. Ideally, a cortisol level should be obtained before therapy, but treatment should never be delayed if there is a strong clinical suspicion of adrenal crisis, which is life-threatening and may present with acute abdominal pain, vomiting, hypotension and hypovolemic shock. Although Addison's disease is rare, it is potentially life-threatening, and there should be a high index of suspicion in patients with nonspecific symptoms but specific physical findings such as hyperpigmentation.

\section{Chantelle Barnard \\ Ronik Kanani \\ Jeremy N. Friedman}

Division of Pediatric Medicine

Department of Pediatrics

University of Toronto Faculty of

Medicine and the Hospital for Sick Children

Toronto, Ont.

\section{References}

1. Betterle C, Dal Pra C, Mantero F, Zanchetta R. Autoimmune adrenal insufficiency and autoimmune polyendocrine syndromes: autoantibodies, autoantigens, and their applicability in diagnosis and disease prediction. Endocr Rev 2002;23:327-64.

2. Arlt W, Allolio B. Adrenal insufficiency. Lancet 2003;361:1881-93.

3. Kong MF, Jeffcoate W. Eighty-six cases of Addison's disease. Clin Endocrinol 1994;41:757-61.

4. Larsen PR, Kronenberg HM, Melmod S, Polonsky KS. Williams textbook of endocrinology. 10th ed. Philadelphia: WB Saunders; 2002. 